\title{
A statistical approach to estimate Global Navigation Satellite Systems (GNSS) receiver signal tracking performance in the presence of ionospheric scintillation
}

\author{
Sreeja Vadakke Veetti1 ${ }^{1, *}$, Marcio Aquino ${ }^{1}$, Luca Spogli ${ }^{2,3}$, and Claudio Cesaroni ${ }^{2}$ \\ Nottingham Geospatial Institute, University of Nottingham, Triumph Road, Nottingham NG7 2TU, UK \\ 2 Istituto Nazionale di Geofisica e Vulcanologia, Via di Vigna Murata 605, 00143 Rome, Italy \\ 3 SpacEarth Technology, Via di Vigna Murata 605, 00143 Rome, Italy
}

Received 30 May 2018 / Accepted 24 September 2018

\begin{abstract}
Ionospheric scintillation can seriously impair the Global Navigation Satellite Systems (GNSS) receiver signal tracking performance, thus affecting the required levels of availability, accuracy and integrity of positioning that supports modern day GNSS based applications. We present results from the research work carried out under the Horizon 2020 European Commission (EC) funded Ionospheric Prediction Service (IPS) project. The statistical models developed to estimate the standard deviation of the receiver Phase Locked Loop (PLL) tracking jitter on the Global Positioning System (GPS) L1 frequency as a function of scintillation levels are presented. The models were developed following the statistical approach of generalized linear modelling on data recorded by networks in operation at high and low latitudes during the years of 2012-2015. The developed models were validated using data from different stations over varying latitudes, which yielded promising results. In the case of mid-latitudes, as the occurrence of strong scintillation is absent, an attempt to develop a dedicated model proved fruitless and, therefore, the models developed for the high and low latitudes were tested for two mid-latitude stations. The developed statistical models can be used to generate receiver tracking jitter maps over a region, providing users with the expected tracking conditions. The approach followed for the development of these models for the GPS L1 frequency can be used as a blueprint for the development of similar models for other GNSS frequencies, which will be the subject of follow on research.
\end{abstract}

Keywords: Global Navigation Satellite Systems / receiver signal tracking performance / statistical modelling / ionospheric scintillation

\section{Introduction}

The effect of the Earth's ionospheric environment currently represents the single largest contributor to the Global Navigation Satellite System (GNSS) error budget and abnormal ionospheric conditions can impose serious degradation on GNSS system functionality, including integrity, accuracy and availability. In particular, small-scale time varying plasma density irregularities in the ionosphere can introduce fluctuations in the amplitude and phase of the received GNSS signal, a phenomena known as scintillation (Kintner et al., 2001). Scintillation occurrence shows large day-to-day variability with dependence on local time, season, latitude, longitude, as well as solar and geomagnetic activity. The global morphology of

\footnotetext{
*Corresponding author: v.sreeja@gmail.com; sreeja.veettil@nottingham.ac.uk
}

ionospheric L-band scintillation occurrence during the solar maximum and minimum conditions is well known (Basu et al., 2002; Kintner et al., 2007; Alfonsi et al., 2011; Prikryl et al., 2015). It has been observed that the two regions where scintillation occurs more predominantly are the equatorial bands extending from about $20^{\circ} \mathrm{N}$ to $20^{\circ} \mathrm{S}$ geomagnetic latitudes and the high latitude regions extending from $65^{\circ}$ to $90^{\circ}$ geomagnetic latitudes. However, in these two regions, the processes that govern the generation of the irregularities causing scintillation are quite different, thereby leading to significant differences in the characteristics of the observed scintillation effects (Jiao \& Morton, 2015).

Global Navigation Satellite System (GNSS) receivers need robust and continuous tracking of the satellite signals in order to compute the distance travelled by the incoming signal as accurately as possible. Demodulation of the navigation data from the incoming signal requires an exact replica of the signal, 
which can be achieved by tracking its phase through a Phase Locked Loop (PLL). However, ionospheric scintillation can degrade the GNSS receiver signal tracking performance. Rapid phase fluctuations associated with scintillation may cause the receiver PLL to lock onto a wrong phase while still tracking the signal, or even to lose lock completely (Humphreys et al., 2005). The receiver PLL performance is usually evaluated in terms of the phase tracking error variance, which is observed to correlate well with the scintillation levels at both high and low latitudes (Sreeja et al., 2012; Aquino \& Sreeja, 2013). Although some high grade receivers, e.g. with an adaptive tracking loop, may be able to maintain track during moderate to strong scintillation conditions, the errors due to scintillation still propagate to the GNSS measurements leading to degradation in the positioning accuracy (Marques et al., 2018). Several efforts have been made to model the effects of scintillation on the Global Positioning System (GPS) receiver PLL performance. These can be summarized as: (i) development of scintillation sensitive PLL tracking models (Conker et al., 2003; Moraes et al., 2014), (ii) evaluation of PLL performance using a GPS signal simulator (Morrissey et al., 2004) and (iii) observation of receiver performance during actual events of scintillation in locations of interest (Groves et al., 2000).

With the growing reliance on GNSS for several modern life applications, including those demanding high positioning accuracy, precise ionospheric forecasts can contribute to the understanding and mitigation of the impact of significant ionospheric related events on our technology-based society. In this context, the research presented here was carried out under the "Ionosphere Prediction Service" (IPS) project funded by the European Commission (EC) under Horizon 2020, in the frame of the Galileo programme. The project is led by Telespazio (Italy) in collaboration with University of Nottingham (UoN, United Kingdom), Istituto Nazionale di Geofisica e Vulcanolgia (INGV, Italy), the University of Tor Vergata (UTOV, Italy), Nottingham Scientific Ltd (NSL, United Kingdom) and Telespazio VEGA (Germany). The main aim of this project is to translate the state of the ionosphere into GNSS user-devoted metrics through the design and development of an ionospheric prediction service prototype. The user-devoted products are tailored to meet the needs of the different communities, namely aviation, mass market, high accuracy and critical infrastructures monitoring, to which the service is targeted. The core scientific contribution of this project is represented by the research activities carried out by the project's research partners, namely UTOV, INGV and UoN, dealing with different topics of Space Weather phenomena from the Sun to the ionosphere affecting GNSS service providers and the user community.

In this framework, statistical models have been developed for the first time by UoN to estimate the standard deviation of the receiver PLL tracking jitter on the GPS L1 frequency separately at high and low latitudes as a function of the scintillation levels - these are presented and discussed. The research does not include the estimation of the scintillation levels, which is a topic being dealt with separately in the IPS project. Therefore the statistical models are developed based on real longterm data that is representative of historical scintillation levels actually observed in the different regions of the globe addressed in the project. The considerable size of the data set (12744760
(2.14 GB) and 11790943 (2.05 GB) data points respectively for the high and low latitudes) and the varied levels of scintillation that it covers provide a large and representative sample to ensure the statistical robustness of the models, which are validated in the research. The data and methodology are described in Section 2, followed by results and discussions in Section 3. Finally, the conclusions are presented in Section 4.

\section{Data and methodology}

The data collected by networks of specialised Ionospheric Scintillation Monitoring Receivers (ISMRs) that were in operation at the high and low latitudes during the years of 2012-2015 have been exploited to develop the statistical models to estimate the standard deviation of the receiver PLL tracking jitter, $\sigma_{\mathrm{PLL}}$, on the GPS L1 frequency. In the development of these models, only measurements from satellites with an elevation angle greater than $20^{\circ}$ were considered, in order to remove the contribution from non-scintillation related effects, such as multipath. Also, a minimum lock time threshold of $240 \mathrm{~s}$ was used to allow the convergence of the phase detrending filter. Two types of ISMRs have been used in this analysis, namely the Novatel GSV4004 and the Septentrio PolaRxS. The geographic latitude and longitude of the stations along with the GNSS receiver type used in the analyses are shown in Table 1. The data availability at the different stations are also shown in Table 1.

Statistical models have been developed to estimate $\sigma_{\mathrm{PLL}}$ as a function of scintillation indices and $\mathrm{ROT}_{\mathrm{rms}}$, the latter defined as the root mean square of the Rate of change of slant Total Electron Content (STEC). Both types of ISMR used in this study use similar algorithms to provide the scintillation indices, namely the amplitude scintillation index, S4 and the phase scintillation index, $\sigma_{\varphi}$. The analyses presented in Sreeja et al. (2011a) show that the scintillation indices recorded by these two types of ISMRs are comparable.

The S4 is defined as the standard deviation of the received signal power normalized by its mean value and $\sigma_{\phi}$ is defined as the standard deviation of the detrended carrier phase using a high pass filter with $0.1 \mathrm{~Hz}$ cut off computed over $60 \mathrm{~s}$. In this study, the one minute $\sigma_{\phi}$ and S4 indices recorded on the GPS L1 frequency per satellite-receiver link are used. The values of the scintillation indices values typically varies between 0 and 1 . The uncalibrated STEC and the differential TEC (dTEC) values computed every $15 \mathrm{~s}$ per satellite-receiver link are also recorded by both types of ISMR (Van Dierendonck et al., 1993; Septentrio PolaRxS application manual, 2010). The dTEC values are computed from the carrier phase measurements and refer to the change in TEC over the four $15 \mathrm{~s}$ intervals during the last minute. The $\mathrm{ROT}_{\mathrm{rms}}$ per satellite-receiver link over a minute used in this study is computed by calculating the root mean square (rms) of the four dTEC values provided by the ISMR over each minute. The development of the models based on $\mathrm{ROT}_{\text {rms }}$ will support the estimation of $\sigma_{\mathrm{PLL}}$ over regions where the scintillation indices or the ISMR data is unavailable, as $\mathrm{ROT}_{\mathrm{rms}}$ can be computed from uncalibrated STEC values that can be estimated using data from any dualfrequency GNSS receiver. The ROT estimation will remove any biases in the STEC measurements due to the differencing 
Table 1. Geographic latitude and longitude of the stations.

\begin{tabular}{lcccccc}
\hline ID & Station & Lat $\left({ }^{\circ} \mathrm{N}\right)$ & Long $\left({ }^{\circ} \mathrm{E}\right)$ & Sector & Receiver type & Data availability \\
\hline NYA & Ny-Ålesund & 78.92 & 11.93 & High latitude & GSV4004 /PolaRxS & $2005-2015$ \\
BRO & Brønnøysund & 65.46 & 12.22 & High latitude & GSV4004 /PolaRxS & $2002-2003 ; 2011-2013$ \\
IQA & Iqaluit & 63.74 & -68.54 & High latitude & GSV4004 & $2012-2015$ \\
CBB & Cambridge Bay & 69.10 & -105.12 & High latitude & GSV4004 & $2012-2015$ \\
NOT & Nottingham & 52.95 & -1.19 & Mid Latitude & GSV4004 & $2012-2015$ \\
CYP & Cyprus & 35.18 & 33.38 & Mid Latitude & PolaRxS & $2012-2015$ \\
PAL & Palmas & -10.12 & -48.18 & Low latitude & PolaRxS & $2012-2015$ \\
POA & Porto Alegre & -30.04 & -51.07 & Low latitude & PolaRxS & $2012-2015$ \\
PRU & Presidente Prudente & -22.07 & -51.24 & Low latitude & PolaRxS & $2012-2015$ \\
\hline
\end{tabular}

of the STEC values over time. The range of $\mathrm{ROT}_{\mathrm{rms}}$ values used in the model development is between 0 and 5 .

As the range of validity of the developed models is driven by the model input data, the distribution of the one minute scintillation indices and $\mathrm{ROT}_{\mathrm{rms}}$ values covered by the four year long data used in the model development is shown in Table 2.

It can be observed from Table 2 that there are no major inherent limitations or bias in the input data, as all levels of scintillation are represented well. Therefore, the developed statistical models can be used under different scintillation conditions. Furthermore, the models are more likely to perform well during weak and moderate scintillation levels characterized by $\mathrm{S} 4 / \sigma_{\phi}<0.7$ and $\mathrm{ROT}_{\text {rms }}<2$, where there is an abundance of input data.

The $\sigma_{\mathrm{PLL}}$ at high latitudes was computed using the scintillation sensitive PLL tracking model of Conker et al. (2003), hereafter referred to as the Conker model. However, this model is limited to weak-to-moderate levels of scintillation, S4 (L1) $<0.707$, and hence cannot be applied for all levels of scintillation. This is particularly relevant for the equatorial and low latitudes, where very strong scintillation conditions are frequently encountered, with S4 (L1) reaching values greater than 0.8 . To overcome this limitation and to enable the computation of $\sigma_{\mathrm{PLL}}$ over the equatorial and low latitudes, even under strong levels of scintillation, the PLL tracking model proposed in Moraes et al. (2014), hereafter referred to as the $\alpha-\mu$ model, was exploited. The parameters required to compute $\sigma_{\text {PLL }}$ using either of these models are recorded by the ISMRs. The Conker model assumes a Nakagami-m distribution for the statistical characterization of amplitude scintillation, thus leading to the limitations in the model. The $\alpha-\mu$ model is an extension of the Conker model and is based on the $\alpha-\mu$ distribution, a more realistic distribution for amplitude scintillation (Moraes et al., 2013). The $\alpha$ and $\mu$ coefficients can be computed either from the field recorded data, provided high rate $50 \mathrm{~Hz}$ data is available, or from an established empirical relationship between $\alpha$ and S4 (Moraes et al., 2014).

Statistical methods in the form of polynomial regression analysis have been applied previously to analyze the correlation of PLL tracking jitter with scintillation levels (Sreeja et al., 2012; Aquino \& Sreeja, 2013). However, these correlation studies were limited to small data sets, usually from a limited number of individual days, not allowing for any attempt to develop robust statistical models that could be reliably used to estimate the receiver signal tracking performance as a function of scintillation levels. In this study, for the first time the statistical approach of Generalized Linear Modelling (GLM) was applied on a long-term, four years long data set, i.e. from 2012 to 2015, covering different seasons, solar and geomagnetic activity conditions, to develop models to estimate $\sigma_{\mathrm{PLL}}$ as a function of scintillation levels.

The GLM (McCullagh \& Nelder, 1989) is a flexible generalization of linear regression models including response variables that do not necessarily have a Gaussian error distribution and uses the maximum likelihood estimation to estimate the model coefficients. The analysis of the error distribution curve for the response variable, $\sigma_{\mathrm{PLL}}$ in this case, showed that they follow a normal distribution, therefore this approach can be considered as linear regression, which is a special case of GLM. The standard errors of the statistical model coefficients are inversely proportional to the square root of the size of the dataset involved in the model development. In this study, using 4 years of data, the developed statistical models have smaller standard errors than previous models (Sreeja et al., 2012; Aquino \& Sreeja, 2013) in the estimated model coefficients, thus resulting in an increased ability to estimate under various conditions. To determine the significance of each coefficient estimated in the model, a Matlab built in statistical test based on the t-statistic was used (Lehmann \& Romano, 2005) at a $5 \%$ significance level, which is commonly used in this type of analysis.

For the validation of the developed models, a data sacrifice strategy was adopted, whereby selected data, covering scintillation and non-scintillation days during different seasons, has been left out of the development phase of the models. The criteria for categorizing a day as scintillation or non-scintillation was based on the temporal profiles of the phase and amplitude scintillation indices for the high and low latitudes respectively. The following criteria was applied to categorize the day as scintillation: (1) the threshold for amplitude and phase scintillation index is set as 0.3 as this study focuses on moderate to strong levels of scintillation; (2) the satellite elevation cut off is set at $20^{\circ}$; (3) the one minute scintillation index values remain above the threshold for more than $15 \mathrm{~min}$; (4) for equatorial and low latitude stations, this 15 min interval refers to the post sunset period and for high latitudes, this 15 min interval refers to any time during the day. Days on which the scintillation index values were less than 0.3 for the whole day were categorized as non-scintillation days. In addition, manual visual inspections of the figures illustrating the temporal profiles of the scintillation indices were performed to confirm the selection of scintillation and non-scintillation days. The goodness of fit of the model estimations were evaluated by using the correlation coefficient, $R$, and the Root Mean 
Table 2. Number of samples in different intervals of scintillation levels at high and low latitudes.

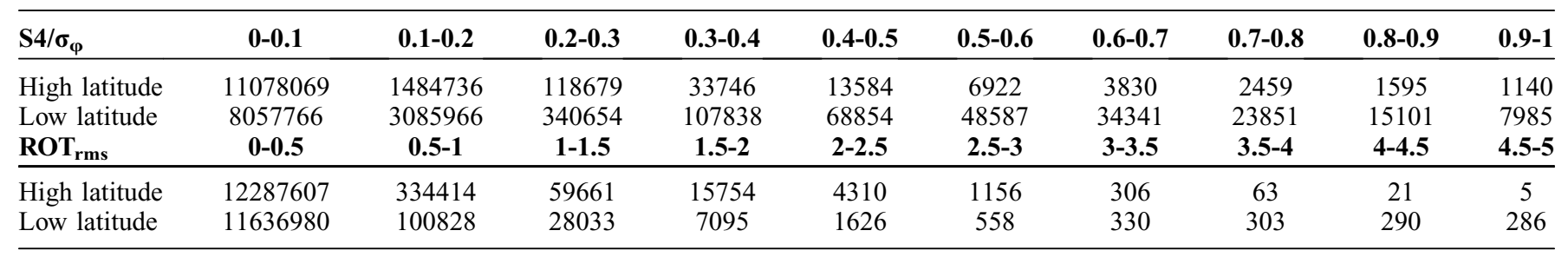

Squared Error (RMSE) of the residuals. $R$ is a measure of how well the model estimates the variability in the observations, whereas the RMSE is a measure of how accurately the model estimates the magnitude of the observations themselves. A higher value for $R$ indicates that the model represents the trend of the observations well while not giving any indication of possible underlying systematic errors, whereas a low value of the RMSE indicates that the model contains no systematic errors. $R$ is relevant under moderate to strong scintillation conditions, as the model must be able to properly recover the peaks due to scintillation occurrence, whereas the RMSE is relevant under any scintillation conditions, as it represents the closeness between the observations and their model estimations. Moreover, under weak scintillation conditions, where peaks in the observations do not usually occur, $R$ becomes less important by comparison to the RMSE, which alone indicates the closeness between the observations and their model estimations. Low RMSE values will indicate more accurate model estimations than high values of $R$ and therefore the former was used in the analyses as the relevant metric to evaluate the goodness of fit.

\section{Results and discussions}

The development of the statistical models following the approach of GLM to estimate $\sigma_{\mathrm{PLL}}$ at high and low latitudes is described in this section. The validation of the developed models is also described.

\subsection{High latitude model}

The data recorded at the high latitude stations, IQA and $\mathrm{CBB}$ in the Canadian High Arctic Ionospheric Network (Jayachandran et al., 2009) were used to develop the statistical models incorporating the dependence on the scintillation indices and the $\mathrm{ROT}_{\text {rms. Scintillation at high latitudes fre- }}$ quently occurs in the cusp region with extension into dayside polar cap and in the nightside auroral oval (Prikryl et al., 2015). This was the rationale behind using data from stations CBB and IQA for model development, which are located in the cusp/polar cap and the auroral oval respectively.

To enable the computation of $\sigma_{\mathrm{PLL}}$ over the high latitudes, the Conker model has been exploited. Using the statistical test described in the previous section, the models developed for the high latitudes showed a dependence of $\sigma_{\mathrm{PLL}}$ on the phase scintillation index, $\sigma_{\varphi}$ as well as on the $\mathrm{ROT}_{\mathrm{rms}}$. The models based on $\sigma_{\varphi}$ and $\mathrm{ROT}_{\mathrm{rms}}$ developed using data from IQA have the following forms, respectively:

$$
\begin{aligned}
\sigma_{\mathrm{PLL}}(\mathrm{mm})= & 3.1246+0.2319 \times \sigma_{\varphi}+1.1296 \times \sigma_{\varphi}^{2} \\
\sigma_{\mathrm{PLL}}(\mathrm{mm})= & 3.0941+0.1452 \times \mathrm{ROT}_{\mathrm{rms}}-0.0226 \\
& \times \mathrm{ROT}_{\mathrm{rms}}^{2}
\end{aligned}
$$

The models based on $\sigma_{\varphi}$ and $\mathrm{ROT}_{\mathrm{rms}}$ developed using data from $\mathrm{CBB}$ have the following forms, respectively:

$$
\begin{aligned}
& \sigma_{\mathrm{PLL}}(\mathrm{mm})=3.11+0.242 \times \sigma_{\varphi}+1.13 \times \sigma_{\varphi}^{2} \\
& \sigma_{\mathrm{PLL}}(\mathrm{mm})= 3.092+0.1463 \times \mathrm{ROT}_{\mathrm{rms}}-0.0111 \\
& \times \mathrm{ROT}_{\mathrm{rms}}^{2} .
\end{aligned}
$$

It can be observed from the above equations that the coefficients of the developed models based on data from IQA and $\mathrm{CBB}$ are quite close to one another, thus indicating that either set of models (Eqs. (1) and (2) or Eqs. (3) and (4)) would be able to represent well the $\sigma_{\mathrm{PLL}}$ over varying latitudes. Therefore, the models developed using data from IQA were chosen as the high latitude models and used in the subsequent analysis. For illustration, a sample result showing the validation of the model represented in equations (1) and (2) on 03 March 2012, a day selected using the data sacrifice strategy described in the previous section, at station IQA is shown respectively in the top and bottom panels of Figure 1. In this figure, the black symbols indicate the observed values of $\sigma_{\mathrm{PLL}}$, whereas the red symbols indicate the model estimations.

The RMSE of the residuals estimated for this validation day was $0.0664 \mathrm{~mm}$ and $0.0504 \mathrm{~mm}$ and the correlation coefficient $\mathrm{R}$ was 0.91 and 0.60 respectively for the models represented in equations (1) and (2), i.e. for the models based on $\sigma_{\varphi}$ and $\mathrm{ROT}_{\text {rms }}$ respectively. This indicated that the models were able to correctly estimate both the values and the variability of the actual observations. The results from the days selected using the data sacrifice strategy described in the previous section for the validation of the models represented in (1) and (2) are summarized in Table 3. The table shows the average correlation coefficient $\mathrm{R}$ and the average RMSE in $\mathrm{mm}$ along with the number of days used for validation.

To further assess the suitability of the models represented in equations (1) and (2) to estimate $\sigma_{\mathrm{PLL}}$ at high latitudes, these models were also tested at the European high latitude stations of BRO and NYA, which did not contribute any data to the model development. The results from the validation days are shown in Table 4.

The fact that the choice of the cut off frequency used in detrending the phase can impact the estimation of the phase 

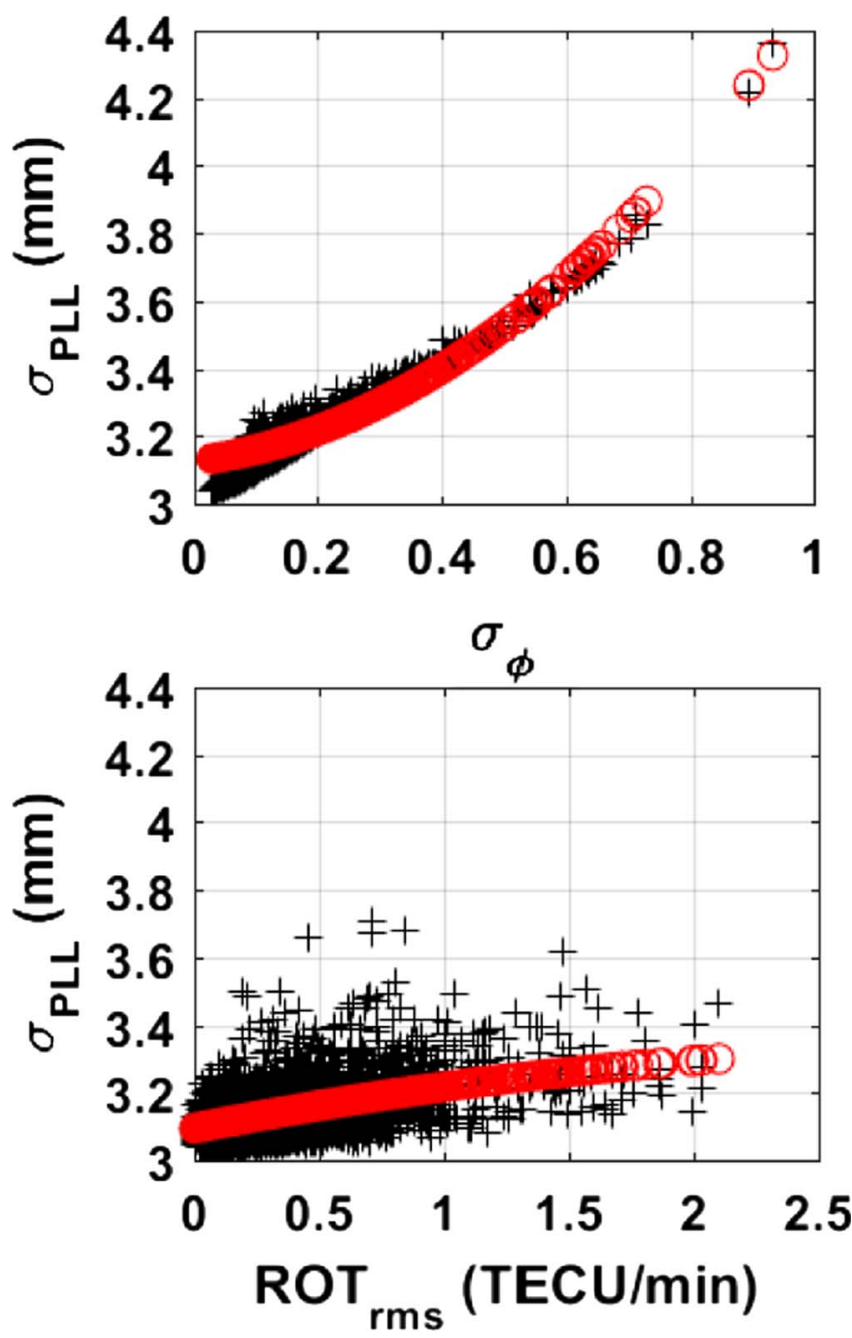

Fig. 1. Variations in the observed (black symbols) and the model estimated (red symbols) $\sigma_{\mathrm{PLL}}$ as a function, respectively, of $\sigma_{\varphi}$ (top) and $\mathrm{ROT}_{\text {rms }}$ (bottom), at the station IQA on 03 March 2012.

scintillation index, $\sigma_{\varphi}$ is known for a number of years (Forte \& Radicella, 2002; Mushini et al., 2012). The $0.1 \mathrm{~Hz}$ cutoff frequency is widely implemented in ISMRs used for scintillation characterization. The results presented in this manuscript are based on data recorded by ISMRs. The impact of the results presented in Wang et al. (2018) could be on the developed high latitude models based on $\sigma_{\varphi}$ (Eqs. (1) and (3)). The assessment of the impact can only be carried out in a future study when a more definitive choice on the detrending cut off frequency is widely accepted by the community and therefore is now beyond the scope of this manuscript. The evidence presented in Wang et al. (2018) will not have an impact on the models based on $\mathrm{ROT}_{\mathrm{rms}}$, as $\mathrm{ROT}_{\mathrm{rms}}$ is computed from uncalibrated STEC values estimated using data from any dual-frequency GNSS receiver.

\subsection{Low latitude model}

The data recorded at station PRU in Brazil was used to develop the statistical model for the low latitudes. This station was chosen because it lies close to the crest of the Equatorial
Ionization Anomaly (EIA), where the occurrence of low latitude scintillation is frequent (Basu et al., 2002). To enable the computation of $\sigma_{\mathrm{PLL}}$ over the equatorial and low latitudes, the $\alpha-\mu$ model has been exploited. Based on the chosen statistical test described in the previous section, the models developed for the low latitudes showed a dependence on the amplitude scintillation index, $\mathrm{S} 4$ and on the $\mathrm{ROT}_{\mathrm{rms}}$. These models have the following forms, respectively:

$$
\begin{gathered}
\sigma_{\mathrm{PLL}}(\mathrm{mm})=3.0761+0.2565 \times \mathrm{S} 4+0.7119 \times \mathrm{S}^{2} \\
\sigma_{\mathrm{PLL}}(\mathrm{mm})=3.0111+0.4828 \times \mathrm{ROT}_{\mathrm{rms}} \\
-0.0326 \times \mathrm{ROT}_{\mathrm{rms}}^{2}
\end{gathered}
$$

The validation results of the models represented in equations (5) and (6) for the station PRU are summarized in Table 5.

As with the high latitude models, the suitability of the models represented in equations (5) and (6) were also tested at the Brazilian low latitude stations POA and PAL, which did not contribute any data to the model development. Table 6 summarizes the validation results for these two stations.

The validation results presented in Tables 3-6 indicate that both the developed high and low latitude models perform well under scintillation and non-scintillation conditions. The average values of $R$ are mostly above 0.5 , indicating correlation between the observed trend and the model estimated trend. The average RMSE values are all below $0.2 \mathrm{~mm}$, which is one order of magnitude smaller than the precision of the GPS L1 carrier phase measurements of about $2 \mathrm{~mm}$. This indicates that the values of the residuals are well below the L1 tracking noise level. Under these conditions, the value of the RMSE is the relevant criterion to evaluate the goodness of fit of the model estimations.

From the above tables, values of $R$ observed at the low latitudes are on average lower than those observed at the high latitudes. This can be attributed to the fact that at low latitudes, during strong scintillation, there could also be fluctuations in the received signal to noise ratio $(\mathrm{CN} 0)$, which have not been taken into account in the development of the above models. The statistical models for the estimation of $\sigma_{\mathrm{PLL}}$ have been developed exploiting their dependence only on the scintillation levels, as these are nowcasted by dedicated algorithms developed by INGV in the context of the IPS project. However, in reality, $\sigma_{\mathrm{PLL}}$ also has a dependence on the $\mathrm{CN} 0$, which is a receiver tracking dependent parameter. Clearly, the models can be further improved if the dependence on this parameter could be incorporated. However, incorporating the dependence on $\mathrm{CN} 0$ in the models is out of the scope of the IPS project, as nowcasted values of $\mathrm{CN} 0$ are not foreseen to be provided by the prototype.

\subsection{Mid-latitude model}

In the case of mid-latitude stations, the occurrence of strong scintillation is almost absent and hence $\sigma_{\mathrm{PLL}}$ values are very low. On the model development at these latitudes, the statistical test described in Section 2 indicated that none of the estimated coefficients were statistically significant, leading to conclude that any attempt using our approach to develop a model specific 
Table 3. Results from the validation days at IQA.

\begin{tabular}{ccccc}
\hline Model & Validation days & Average correlation coefficient, $R$ & Average RMSE (mm) & Number of validation days \\
\hline Equation (1) & Scintillation & 0.89 & 0.0607 & 41 \\
Equation (1) & Non-scintillation & 0.87 & 0.0649 & 41 \\
Equation (2) & Scintillation & 0.61 & 0.0526 & 41 \\
Equation (2) & Non-scintillation & 0.56 & 0.0441 & 41 \\
\hline
\end{tabular}

Table 4. Results from the validation days at BRO and NYA.

\begin{tabular}{llcccc}
\hline Station & Model & Validation days & Average correlation coefficient, $R$ & Average RMSE (mm) & Number of validation days \\
\hline BRO & Equation (1) & Scintillation & 0.78 & 0.1048 & 37 \\
BRO & Equation (1) & Non-scintillation & 0.76 & 0.0799 & 37 \\
BRO & Equation (2) & Scintillation & 0.58 & 0.1267 & 37 \\
BRO & Equation (2) & Non-scintillation & 0.56 & 0.0808 & 37 \\
NYA & Equation (1) & Scintillation & 0.93 & 0.0622 & 55 \\
NYA & Equation (1) & Non-scintillation & 0.85 & 0.0725 & 55 \\
NYA & Equation (2) & Scintillation & 0.74 & 0.0488 & 55 \\
NYA & Equation (2) & Non-scintillation & 0.70 & 0.0421 & 55 \\
\hline
\end{tabular}

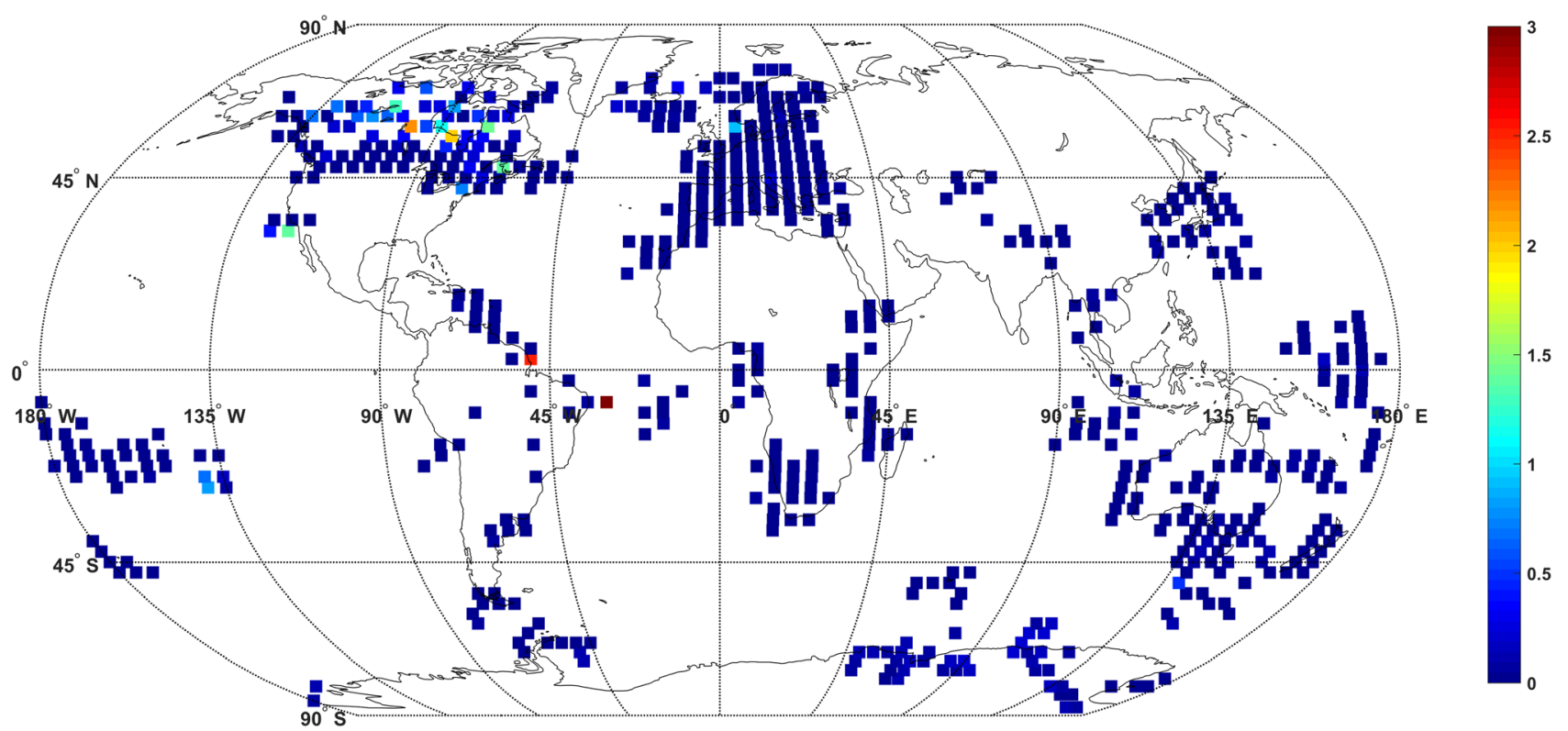

Fig. 2. Nowcasted proxy scintillation indices on a global scale at 05:15 UT on 17 February 2018. PSI values in TECu/min are defined in the color bar.

for the mid-latitudes would be meaningless. Nevertheless, during weak scintillation periods at the mid-latitude station of CYP, the variations in S4 are more pronounced than the variations in $\sigma_{\varphi}$ (Vadakke Veettil et al., 2017) Therefore, the models developed for the low latitudes were tested at this station. Concurrently, at the European mid-latitude station NOT, during weak scintillation periods the variations in $\sigma_{\varphi}$ are more pronounced than the variations in S4 (Aquino et al., 2005). Therefore, the models developed for the high latitudes were tested at this station. The results are summarized in Table 7.

The results shown in Table 7 indicate that the low and high latitude models can be used to estimate $\sigma_{\mathrm{PLL}}$ respectively at the mid-latitude stations CYP and NOT. It is to be noted that at both CYP and NOT stations, the occurrence of strong scintillation was observed to be almost absent, rendering impossible to categorize the days between scintillation and non-scintillation based on the criteria described in Section 2. Also, $\sigma_{\mathrm{PLL}}$ values are much smaller in these regions and therefore most of the time not likely to represent information that might be of relevance for users, as significant peaks are extremely rare. Additionally, it can be observed from Table 7 that the average RMSE values are less than $0.2 \mathrm{~mm}$ and the average $R$ values are mostly above 0.5 , thus indicating that the high and low latitude models may be used to estimate $\sigma_{\mathrm{PLL}}$ respectively at NOT 


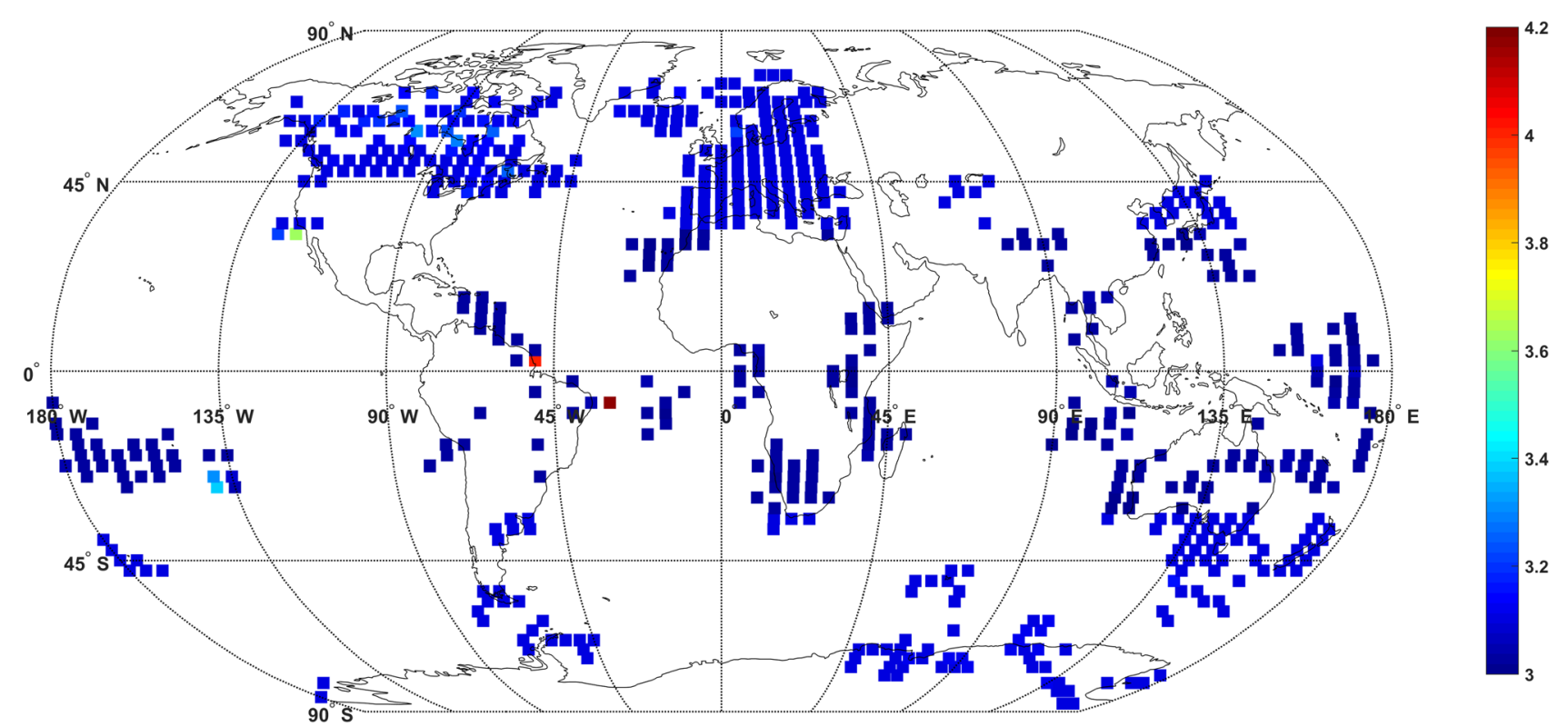

Fig. 3. Nowcasted $\sigma_{\mathrm{PLL}}$ on a global scale at 05:15 UT on 17 February 2018. $\sigma_{\mathrm{PLL}}$ values in mm are defined in the color bar.

Table 5. Results from the validation days at PRU.

\begin{tabular}{ccccc}
\hline Model & Validation days & Average correlation coefficient, $R$ & Average RMSE (mm) & Number of validation days \\
\hline Equation (5) & Scintillation & 0.72 & 0.1128 & 48 \\
Equation (5) & Non-Scintillation & 0.64 & 0.0618 & 48 \\
Equation (6) & Scintillation & 0.66 & 0.1186 & 48 \\
Equation (6) & Non-Scintillation & 0.68 & 0.1000 & 48 \\
\hline
\end{tabular}

Table 6. Results from the validation days at POA and PAL.

\begin{tabular}{llcccc}
\hline Station & Model & Validation days & Average correlation coefficient, R & Average RMSE (mm) & Number of validation days \\
\hline POA & Equation (5) & Scintillation & 0.63 & 0.0562 & 12 \\
POA & Equation (5) & Non-scintillation & 0.57 & 0.0382 & 12 \\
POA & Equation (6) & Scintillation & 0.55 & 0.0923 & 12 \\
POA & Equation (6) & Non-scintillation & 0.51 & 0.0858 & 12 \\
PAL & Equation (5) & Scintillation & 0.71 & 0.0661 & 12 \\
PAL & Equation (5) & Non-scintillation & 0.64 & 0.0375 & 12 \\
PAL & Equation (6) & Scintillation & 0.59 & 0.1057 & 12 \\
PAL & Equation (6) & Non-scintillation & 0.55 & 0.0923 & 12 \\
\hline
\end{tabular}

and CYP. However, further analyses would be necessary to determine a latitude threshold to guide the decision as to what model to apply.

If scintillation information can be nowcasted, the above developed models for the high and low latitudes can be used to nowcast $\sigma_{\mathrm{PLL}}$ on a regional or a global scale and thus used to generate tracking jitter maps (Sreeja et al., 2011b) over a region, which can provide GNSS users with the expected tracking conditions under scintillation. In the scope of the IPS project, partners INGV have developed algorithms to nowcast the scintillation and Proxy Scintillation Indices (PSI), which can then be used as inputs to the above developed models to generate $\sigma_{\mathrm{PLL}}$ maps. As an example, Figure 2 shows the nowcasted PSI values at a global scale on a regular grid of $2.5^{\circ} \times 5^{\circ}$ respectively in latitude and longitude. The map is for 17 February 2018 at 05:15 UT. The PSI values in TECu/ min are defined in the color bar.

The nowcasted $\sigma_{\text {PLL }}$ map, estimated on a global scale over the same grid size as that of Figure 2, using the models represented in equations (2) and (6), for the high and low latitudes respectively, is shown in Figure 3. $\sigma_{\mathrm{PLL}}$ values in $\mathrm{mm}$ are defined in the color bar.

It can be observed from Figures 2 and 3 that regions with higher values of PSI are in correspondence with the occurrence 
Table 7. Results from the validation days at CYP and NOT.

\begin{tabular}{llccc}
\hline Station & Model & Average correlation coefficient, $R$ & Average RMSE (mm) & Number of validation days \\
\hline CYP & Equation (5) & 0.63 & 0.0998 & 32 \\
CYP & Equation (6) & 0.54 & 0.1430 & 32 \\
NOT & Equation (1) & 0.94 & 0.1267 & 24 \\
NOT & Equation (2) & 0.70 & 0.1382 & 24 \\
\hline
\end{tabular}

of higher values of $\sigma_{\mathrm{PLL}}$ according to the model. This kind of map will help in providing the GNSS users with the receiver signal tracking conditions under scintillation. If this type of map with a higher resolution can be constructed over a region, they may then be used to help mitigate the effects of ionospheric scintillation on GNSS positioning. This can be done in association with the strategy introduced in Aquino et al. (2009) based on modifying the least squares stochastic model used for position estimation by assigning weights to individual satellite receiver links.

\section{Conclusion}

The development and validation of statistical models to estimate the receiver $\sigma_{\mathrm{PLL}}$ for the GPS L1 signal developed by UoN under the EC funded IPS project have been presented. The GLM technique was applied to develop the statistical models to estimate $\sigma_{\mathrm{PLL}}$ as a function of the scintillation levels given by the conventional phase and amplitude scintillation

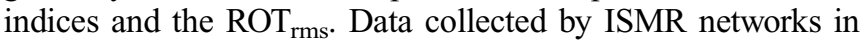
operation at the high and low latitudes during the years of 2012-2015 was exploited to develop these models. The high latitude model showed a dependence on the phase scintillation index, whereas the low latitude model showed a dependence on the amplitude scintillation index. Also, statistical models were developed to incorporate the dependence of $\sigma_{\mathrm{PLL}}$ on $\mathrm{ROT}_{\mathrm{rms}}$ at both high and low latitudes. The validation of the developed models was carried out at different stations covering a range of latitudes, which yielded results that indicate the models' suitability to estimate $\sigma_{\mathrm{PLL}}$ over that range of latitudes. In the case of the mid-latitudes, as the occurrence of strong scintillation is almost absent, an attempt to develop a dedicated model proved fruitless. Nevertheless, to address the mid-latitudes, the models developed for the high and low latitudes were tested, respectively, at two stations in that region, namely Nottingham $\left(53^{\circ} \mathrm{N}\right)$ and Cyprus $\left(37^{\circ} \mathrm{N}\right)$. The rationale is that for these two stations the scintillation characteristics are somewhat similar to what is observed at high and low latitudes respectively. The results of the validation tests indicated that the developed high and low latitude models may be used to successfully estimate $\sigma_{\mathrm{PLL}}$ at mid-latitudes, but more detailed data analyses would be necessary to determine a latitude threshold to guide the decision as to what model to apply. The validation results for the high, mid and low latitude stations indicated average RMSE values below $0.2 \mathrm{~mm}$ and average $R$ values above 0.5 , thus suggesting that the developed models perform well under scintillation and non-scintillation conditions.

Acknowledgements. The research activities related to this paper at the Nottingham Geospatial Institute, University of Nottingham were funded by the EC funded IPS project [contract number: 434/PP/GRO/RCH/15/8381]. This work was supported by the Engineering and Physical Sciences Research Council [Grant Number EP/H003479/1], data from CYP, BRO and NOT stations are from a network of GNSS receivers established through the EPSRC research grant. Data from the PRU, POA and PAL stations in Brazil are part of the CIGALA/CALIBRA network. Monitoring stations from this network were deployed in the context of the Projects CIGALA and CALIBRA, both funded by the EC in the framework of the FP7-GALILEO-2009-GSA and FP7-GALILEO2011-GSA-1a, respectively, and FAPESP Project Number 06/04008-2. Data from NYA station is from the network maintained by INGV. Authors thank Dr. Vincenzo Romano, Dr. Giorgiana De Franceschi, Dr. Lucilla Alfonsi and Dr. Ingrid Hunstad for the valuable support in maintaining the infrastructure and ensuring the quality of the NYA data. The editor thanks Ryan McGranaghan and an anonymous referee for their assistance in evaluating this paper.

\section{References}

Alfonsi L, Spogli L, De Franceschi G, Romano V, Aquino M, Dodson A, Mitchell CN. 2011. Bipolar climatology of GPS ionospheric scintillation at solar minimum. Radio Sci 46: 05. DOI: $10.1029 / 2010 R S 004571$.

Aquino M, Moore T, Dodson A, Waugh S, Souter J, Rodrigues F. 2005. Implications of ionospheric scintillation for GNSS users in Northern Europe. J Navig 58(2): 241-256. DOI: 10.1017/ S0373463305003218.

Aquino M, Monico JFG, Dodson AH, Marques H, De Franceschi G, Alfonsi L, Romano V, Andreotti M. 2009. Improving the GNSS positioning stochastic model in the presence of ionospheric scintillation. J Geod 83: 953-966. DOI: 10.1007/s00190-009-0313-6.

Aquino M, Sreeja V. 2013. Correlation of scintillation occurrence with interplanetary magnetic field reversals and impact on Global Navigation Satellite System receiver tracking performance. Space Weather 11(5): 219-224. DOI: 10.1002/swe.20047.

Basu S, Groves KM, Basu Su, Sultan PJ. 2002. Specification and forecasting of scintillations in communication/navigation links: current status and future plans. J Atmos Solar-Terr Phys 64(16): 1745-1754. DOI: 10.1016/S1364-6826(02)00124-4.

Conker RS, El Arini MB, Hegarty CJ, Hsiao T. 2003. Modeling the effects of ionospheric scintillation on GPS/SBAS availability. Radio Sci 38(1): 1001. DOI: 10.1029/2000RS002604.

Forte B, Radicella SM. 2002. Problems in data treatment for ionospheric scintillation measurements. Radio Sci 37(6): 1096. DOI: $10.1029 / 2001 \mathrm{RS} 002508$.

Groves KM, Basu S, Quinn JM, Pedersen TR, Falinski K, Brown A, Silva R, Ning P. 2000. A comparison of GPS performance in a scintillating environment at Ascension Island. Proceedings of ION GPS 2000, Institute of Navigation, 672-679. 
Marques HA, Silva Marques HA, Aquino M, Vadakke Veettil S, Monico JFG. 2018. Accuracy assessment of Precise Point Positioning with multi-constellation GNSS data under ionospheric scintillation effects. J Space Weather Space Clim 8: A15. DOI: $10.1051 / \mathrm{swsc} / 2017043$.

Humphreys TE, Psiaki ML, Ledvina BM, Kintner PM. 2005. GPS carrier tracking loop performance in the presence of ISs. In: Proceedings of ION GNSS 2005, Institute of Navigation, Long Beach, CA, September, 156-167.

Jayachandran PT, Langley RB, MacDougall JW, Mushini SC, Pokhotelov D, Hamza AM, Mann IR, Milling DK, Kale ZC, Chadwick R, Kelly T, Danskin DW, Carrano CS. 2009. The Canadian high arctic ionospheric network (CHAIN). Radio Sci 44(1): RS0A03. DOI: 10.1029/2008RS004046.

Jiao Y, Morton YT. 2015. Comparison of the effect of high-latitude and equatorial ionospheric scintillation on GPS signals during the maximum of solar cycle 24. Radio Sci 50: 886-903. DOI: $10.1002 / 2015 R S 005719$.

Kintner PM, Kil H, Beach TL, de Paula ER. 2001. Fading timescales associated with GPS signals and potential consequences. Radio Sci 36(4): 731-743. DOI: 10.1029/1999RS002310.

Kintner PM, Ledvina BM, de Paula ER. 2007. GPS and ionospheric scintillations. Space Weather 5(9): S09003, DOI: 10.1029/ 2006SW000260.

Lehmann EL, Romano JP. 2005. Testing Statistical Hypotheses, 3rd ed., Springer, New York, ISBN 0-387-98864-5.

McCullagh P, Nelder J. 1989. Generalized Linear Models. Chapman and Hall/CRC, New York.

Moraes AO, de Paula ER, Perrella WJ, Rodrigues FS. 2013. On the distribution of GPS signal amplitudes during the low-latitude ionospheric scintillation. GPS Solut 17(4): 499-510. DOI: 10.1007/s10291-012-0295-3.

Moraes AO, Costa E, de Paula ER, Perrella WJ, Monico JFG. 2014. Extended ionospheric amplitude scintillation model for GPS receivers. Radio Sci 49(5): 315-333. DOI: 10.1002/2013RS005307.

Morrissey TN, Shallberg KW, Van Dierendonck AJ, Nicholson MJ. 2004. GPS receiver performance characterization under realistic ionospheric phase scintillation environments. Radio Sci 39(1): RS1S20. DOI: 10.1029/2002RS002838.

Mushini SC, Jayachandran PT, Langley RB, MacDougall JW, Pokhotelov D. 2012. Improved amplitude- and phase-scintillation indices derived from wavelet detrended high-latitude GPS data. GPS Solut 16(3): 363-373. DOI: 10.1007/s10291-011-0238-4.

Prikryl P, Jayachandran PT, Chadwick R, Kelly TD. 2015. Climatology of GPS phase scintillation at northern high latitudes for the period from to 2013. Ann Geophys 33: 531-545, DOI: 10.5194/ angeo-33-531-2015.

Septentrio PolaRxS. 2010. Septentrio PolaRxS application manual version 1.0.0.

Sreeja VV, Aquino M, Forte B, Elmas Z, Hancock C, et al. 2011a. Tackling ionospheric scintillration threat to GNSS in Latin America. J Space Weather Space Clim 1: A05. DOI: 10.1051/swsc/2011005.

Sreeja V, Aquino M, Elmas ZG. 2011b. Impact of ionospheric scintillation on GNSS receiver tracking performance over Latin America: Introducing the concept of tracking jitter variance maps. Space Weather 9(10): 10002. DOI: 10.1029/2011SW000707.

Sreeja V, Aquino M, Elmas ZG, Forte B. 2012. Correlation analysis between ionospheric scintillation levels and receiver tracking performance. Space Weather 10(6): S06005. DOI: 10.1029/ 2012SW000769.

Vadakke Veettil S, Haralambous H, Aquino M. 2017. Observations of quiet-time moderate midlatitude L-band scintillation in association with plasma bubbles. GPS Solut 21: 1113. DOI: 10.1007/ s10291-016-0598-x.

Van Dierendonck AJ, Klobuchar J, Hua Q. 1993. Ionospheric scintillation monitoring using commercial single frequency $\mathrm{C} / \mathrm{A}$ code receivers. In: Proceedings of ION GPS 1993, Institute of Navigation, pp. 1333-1342.

Wang Y, Zhang Q-H, Jayachandran PT, Moen J, Xing Z-Y, Chadwick R, Ma Y-Z, Ruohoniemi JM, Lester M. 2018. Experimental evidence on the dependence of the standard GPS phase scintillation index on the ionospheric plasma drift around noon sector of the polar ionosphere. J Geophys Res Space Phys 123: 2370-2378. DOI: 10.1002/2017JA024805.

Cite this article as: Vadakke Veettil S, Aquino M, Spogli L \& Cesaroni C 2018. A statistical approach to estimate Global Navigation Satellite Systems (GNSS) receiver signal tracking performance in the presence of ionospheric scintillation. J. Space Weather Space Clim. 8, A51. 